\section{Bone Autofluorescence and Mineral Content}

WHEN ground bone sections are examined microscopically using an ultra-violet light source, some of the osteons autofluoresce with less intensity than others. By reference to tetracycline-marked osteons, it can be shown that the less-fluorescent osteons are those most recently formed.

Microradiographs of ground bone sections also reveal that the more recently formed osteons contain less mineral than older ones.

It seems necessary to correlate these two findings on a single specimen of bone before assuming that the weakly fluorescent and poorly calcified osteons are the same.

A thin transverse section from an osteoporotic femur was cut unfixed and unembedded. This was ground to about $75 \mu$ thickness between ground-glass plates. A contact microradiograph of the specimen was taken ( $3 \mathrm{~min}$ exposure at $20 \mathrm{kV} 200 \mu \mathrm{amp}$ ). The same specimen was then mounted and a photograph of the autofluoresce taken (200-amp mercury vapour lamp, Reichert exciter filter $E$ I, dark ground condenser and $S p$ I absorption filter).

\title{
BIOLOGY
}

\section{Life-cycle of Cyathostoma lari Blanchard, 1849 (Nematoda, Strongyloidea)}

AN investigation is at present proceeding into helminth parasitism in the herring gull (Larus argentatus Pontopp.) population of North Caernarvonshire and Anglesey, particularly of the Newborough Warren Nature Reserve, where a scheme to limit the numbers of birds is in operation. One parasite that is commonly found, particularly among juvenile birds, is Cyathostoma lari Blanchard, 1849 , a species first described in Britain by Burt and Eadie ${ }^{1}$, and more recently recorded by Pemberton ${ }^{2-4}$.

Little is known about the life-cycles of members of the genus Cyathostoma, but Pemberton ${ }^{2}$ recorded the experimental infection of a domestic fowl with $C$. lari; the bird became infected only when the nematode larvae were first passed through an earthworm as intermediate host. Larval development in the intermediate host was not described.

Autofluorescent photograph

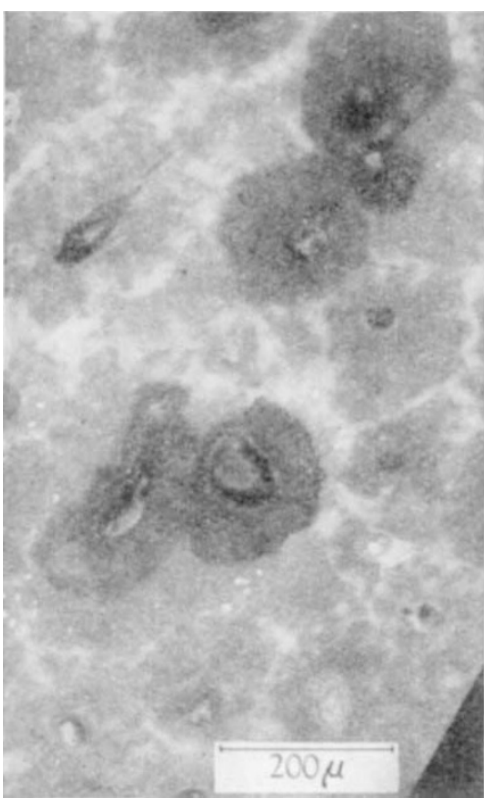

$A$
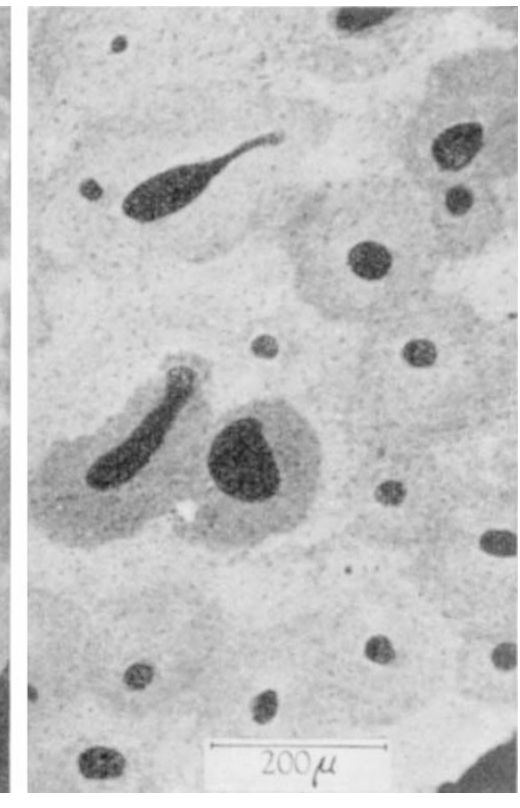

B

Fig. 1

A comparison of the photographs (Fig. $1 A$ and $B$ ) reveals that where the bone is most densely mineralized there is maximum autofluorescence.

However, the difference in intensity of autofiuorescence within a ground section of bone is not due directly to the mineral content, as can be shown by demineralizing it in either EDTA or acid, when the autofluorescence remains unchanged.

The intensity of autofluorescence of bone is altered by prolonged fixation in formalin so that the 'new' osteons frequently show more fluorescence than the background bone. The foregoing findings do not, therefore, necessarily hold true in formalin-fixed tissue.

I thank Mr. R. V. Ely of the M.R. Research Trust for taking the microradiograph.

A. I. D. Prentice

Black Notley Hospital, Braintree, Essex.
Very few larvae of $C$. lari can be recovered from earthworms even where they are fed eggs or larvae of the nematode. Furthermore, very few adult nematodes ever develop if infected earthworms are fed to herring gulls or domestic chicks. Experiments on the development of $C$. lari larvae in vitro and in the earthworm were therefore undertaken.

Eggs of $C$. lari were allowed to develop at room temperature $\left(16^{\circ} \mathrm{C} \pm 2^{\circ} \mathrm{C}\right)$. After 5 days larvae had developed within the eggs; 4 days later larva started to hatch, having ecdysed and retained the cast skin of the first larval stage as a sheath. These second stage larvae moved sluggishly and exhibited no migratory movements. These experiments support the results obtained by Pemberton ${ }^{2}$, although the larvae developed and hatched rather more quickly.

Eggs failed to develop when placed in an incubator at $37^{\circ} \mathrm{C}$ either immediately after removal from the female worm or after standing in physiological saline at room temperature for up to 4 days. However, eggs kept at $28^{\circ} \mathrm{C}$ developed rapidly and the first larvae hatched after 7 days, but even then many failed to develop. Eggs maintained at room temperature $\left(16^{\circ} \mathrm{C} \pm\right.$ $2^{\circ}$ C) began to hatch after 9 days and by the twentieth day twice as many larvae had emerged than in the case of the eggs kept at $28^{\circ} \mathrm{C}$. Eggs kept at $4^{\circ} \mathrm{C}$ for 4 days failed to develop when later left at room temperature. It would appear, therefore, that a period of development at a comparatively low but not freezing temperature is necessary in the development of this nematode.

If, however, the eggs are kept in physiological saline containing penicillin and streptomycin (300 units $/ \mathrm{ml}$. and $0.3 \mathrm{mg} / \mathrm{ml}$. respectively) for a minimum period of 17 days at room temperature and then placed in an incubator at $37^{\circ} \mathrm{C}$, there is a mass hatching of larvae 1-2 days later and these larvae move more actively than those kept at $16^{\circ} \mathrm{C}$.

Fggs and larvae kept at room temperature for 23 days were fed to each of three 15-day-old domestic fowl chicks. Twenty-one days later the chicks were killed and $C$. lari were found in all of them. In this instance the life-cycle from bird to bird has been completed in 44 days, without the use of an intermediate host. It would appear therefore, that the earthworm may act as a paratenic host, not as a true intermediate host, and it may be that it acts as a reservoir of infection for $C$. lari during the cold weather. 